\title{
La actual restructuración del sistema financiero de la Zona Euro
}

The recent prudential reform to the banking system of the Eurozone

\section{Valeria Ivett Luna Flores*}

\section{Resumen}

El principal objetivo del presente artículo es dar a conocer las tres principales funciones de la Unión Bancaria Europea para poder comprender mejor las medidas de regulación prudencial que la Zona Euro está considerando a partir del año 2014 y que pueden llegar a ser un ejemplo para los países de América Latina por las reglas aplicadas y el contexto de este nuevo esquema regulatorio.

Palabras clave:

- Banco Central

- Regulación Prudencial

- Sistema Bancario

\section{Abstract}

This document describes the three principal tasks of the European Banking Union, to understand better the measures of prudential regulation applied in the Eurozone since 2014. These new measures could be an example for the Latin American countries due to the rules applied and the new regulatory and supervisory framework implemented.

Keywords:

- Central Bank

- Prudential Regulation

- Banking System

JEL: E58, G28, G21

Ante la recesión de los años 2007-2009 y la crisis de deuda soberana de los países europeos de los años 2010-2012, la iniciativa de la Comisión Europea fue replantear la política de supervisión financiera y de transparencia para asegurar una mayor coordinación a nivel europeo. Por este motivo aparecieron dos nuevas instancias en materia de supervisión bancaria en Europa, la primera fue la Autoridad Bancaria Europea cuya sede está en Londres y empezó a funcionar el $1^{\circ}$ de enero de 2011 y la segunda institución es la Unión Bancaria Europea que asumió sus funciones en noviembre de 2014 y su sede está en Alemania en el BCE.

El objetivo de este artículo es el de presentar cada una de las funciones de la Unión Bancaria Europea en tres secciones. La sección I tratará el tema de la supervisión del sistema bancario a través del mecanismo de supervisión único (MSU), en la sección II se presenta el nuevo esquema que fue diseñado para encontrar una solución lo más pronto posible ante la quiebra de un banco por medio del mecanismo de resolución único (MRU) y la tercera función de esta nueva institución será la de garantizar los depósitos bancarios de los ahorradores mediante la aplicación de un sistema armonizado de garantía de depósitos, esta función se presenta en la sección III. Asimismo, se presentan las principales conclusiones al final del artículo.

* Estudiante de la especialización en Economía Monetaria y Financiera, UNAM. Titular de un DEA y Doctorado en Economía Bancaria por la Universidad de Paris X, Nanterre.Email: luflovaleria@gmail.com. 


\section{Supervisión prudencial del sistema bancario de la Zona Euro}

La Autoridad Bancaria Europea (EBA) está encargada de definir el contenido de las reglamentaciones financieras, de realizar las pruebas de estrés (stress test) y de garantizar que las diferentes reglamentaciones financieras de los 28 países de la Unión Europea sean similares.

Dentro de la amplia gama de funciones del Banco Central Europeo, una nueva función que le ha sido asignada a partir del 4 de noviembre de 2014 ha sido la de encargarse de la supervisión prudencial mediante un nuevo proyecto que empezó en junio de 2012 pero fue aprobado hasta el 15 de abril de 2014 por el Parlamento Europeo, que comprende una nueva arquitectura institucional denominada Unión Bancaria Europea.

Se trata de un nuevo esquema de supervisión diseñado para los países de la Zona Euro, en el cual van a poder participar, en cualquier momento, los países de la Unión Europea que hayan conservado su moneda nacional. La Autoridad Bancaria Europea se encargará de la supervisión prudencial de los países de la Unión Europea que no están participando en el esquema de supervisión de la Unión Bancaria y seguirá cooperando estrechamente con la Unión Bancaria Europea.

La Comisión Europea deseaba que el BCE supervisara directamente a todos los bancos comerciales de la Unión Europea pero dado que el gobierno alemán quería seguir teniendo controladas a las cajas alemanas de ahorro, se decidió otra solución donde el BCE sólo va a supervisar en directo a los más grandes bancos comerciales de la Zona Euro: un total de 130 bancos que representan $82 \%$ de los activos de la Zona Euro, se trata de bancos con un volumen de activos superior a 30 mil millones de euros.

El 4 noviembre del año 2014 entró en vigor el mecanismo de supervisión único (MSU) de la Unión Bancaria que funcionará por medio de un consejo de supervisión único. La Unión Bancaria supervisará que se respeten los niveles de fondos propios y nivel de liquidez de los 130 bancos o grupos bancarios bajo su supervisión y podrá imponer niveles más altos de fondos propios. Si hay incumplimiento con respecto al nivel de fondos propios podrá imponer multas y sanciones administrativas a los bancos.

A pesar de que este mecanismo entró en aplicación en noviembre, ya desde enero de 2014 los bancos comerciales europeos tuvieron que pasar por una revisión de la calidad de sus activos, la "Asset quality review" (AQR), supervisada por el BCE. Un total de 130 establecimientos bancarios fueron supervisados 
Economía Informa núm. 389 noviembre - diciembre • 2014 " " " " " " " " " " -

para conocer su estado de solidez financiera. Así se han identificado los problemas y los riesgos de los balances de los bancos.

El 26 de octubre de 2014 se publicaron los principales resultados: 105 bancos pasaron las pruebas de estrés pero hay 25 bancos europeos que no cumplen con el nivel suficiente de fondos propios. Los resultados de esta revisión de activos servirán de apoyo al consejo de supervisión único para empezar sus funciones. En este examen del BCE participaron los 18 países de la Zona Euro.

\section{Cuadro I}

\section{Zona Euro: resultados de la prueba de revisión de activos}

Número de bancos que participaron:

Número de bancos con fondos propios insuficientes:

Tiempo para encontrar una solución:

Fuente: http://www.ecb.europa.eu/
130

se analizaron todos los activos bancarios que entran dentro del balance de un banco al 31 de diciembre de 2013.

\section{5}

dos semanas para presentar un plan de recapitalización y habrá tolerancia de entre 6 y 9 meses para mejorar el nivel de solvencia. Se revisará si las medidas adoptadas por los bancos comerciales son suficientes o no.

Las autoridades nacionales seguirán supervisando a los bancos comerciales nacionales que no supervise en directo el BCE y colaborarán con el BCE para realizar la supervisión de los grandes bancos nacionales. Sin embargo, aún si el BCE no supervisa en directo a los bancos nacionales pequeños sí podrá intervenir si lo considera necesario.

Al mismo tiempo que el BCE realizaba estas pruebas de estrés y la revisión de los activos financieros de los países de la Zona Euro, la Autoridad Bancaria Europea realizó las pruebas de estrés para un conjunto de 124 bancos de los 28 países de la Unión Europea. La Autoridad Bancaria Europea y el BCE dieron a conocer los resultados de estas pruebas de estrés por país y por banco en sus sitios de internet. Para poder realizar esta revisión fue necesario homologar los conceptos relacionados con los activos de los balances financieros.

Fue la Autoridad Bancaria Europea la institución que diseño la metodología de las pruebas de estrés donde se aplicaron los escenarios que fueron propuestos por el Comité Europeo de Riesgo Sistémico (CERS) con la ayuda de las autoridades nacionales competentes y el BCE. El BCE estima que 25 bancos europeos que no pasaron las pruebas de estrés deben reforzar su nivel de fondos propios. 
La mayor parte de los bancos que no pasaron las pruebas de estrés son bancos de Italia, Grecia, Chipre y Portugal. Una de las ratios de solvencia empleadas en este examen de la calidad de los activos fue la "Core tier one", que relaciona el nivel de fondos propios y de reservas con activos ponderados de acuerdo al nivel de riesgo.

\section{Cuadro 2}

\section{Zona Euro bancos que reprobaron la prueba de estrés}

\begin{tabular}{|c|c|c|}
\hline País & $\begin{array}{l}\text { Número } \\
\text { de Bancos }\end{array}$ & Principales Bancos \\
\hline Italia & 9 & $\begin{array}{l}\text { Banco Popolare di Milano, Banca Popolare di Vicenza, MPs, } \\
\text { Banca Carrige, entre otros }\end{array}$ \\
\hline Chipre & 3 & Bank of Cyprus \\
\hline Grecia & 3 & $\begin{array}{l}\text { Hellenic Bankm National Bank of Greece, Eurobank, Pyraeus } \\
\text { Bank }\end{array}$ \\
\hline Portugal & 1 & Banco Comercial Portugués, es uno de los bancos más grandes \\
\hline Bélgica & 2 & AXA Bank y Dexia \\
\hline Francia & 1 & Caisse de Refinancement de l'Habitat \\
\hline Alemania & 1 & Münchener Hypothekenbank \\
\hline Austria & 1 & Österreichische Volksbanken \\
\hline Irlanda & 1 & Permanent tsb plc \\
\hline Eslovenia & 2 & $\begin{array}{l}\text { El principal banco de Eslovenia "Nova Ljubljanska Banka" y } \\
\text { Nova Kreditna Banka Maribor }\end{array}$ \\
\hline
\end{tabular}

De los 15 bancos españoles analizados en esta prueba no hubo ninguno que tuviera que ser recapitalizado. Los bancos españoles que participaron fueron: Banco BBvA, Sabadell, BFA-Bankia, Banco Mare Nostrum (BмN), Popular, Santander, Bankinter, Ibercaja Banco, CaixaBank, Catalunya Banc, Banco de Crédito Cooperativo-Cajamar, Kutxabank, Liberbank, Unicaja Banco y Abanca.

Fuente: Elaboración propia con datos publicados en el periódico "Le Monde", noviembre 2014.

Dado que este examen de la revisión de los activos fue hecho basándose en las cifras publicadas el 31 de diciembre de 2013, durante el año 2014 ha habido algunos bancos que han podido aumentar su capital. Los bancos pueden aumentar sus fondos propios si logran aumentar su producto neto bancario o bien gracias a un aumento de capital.

Así, los bancos de la Zona Euro continúan manteniendo numerosos préstamos de baja calidad y tienen que seguir una política de crédito estricta cuando se trata de otorgar nuevos créditos, para no acumular malos préstamos complementarios. Es por esto que la Unión Bancaria implica primeramente una supervisión unificada de los bancos, dado que la falta de rigor ha frenado el saneamiento del sector bancario. 
Economía Informa núm. 389 noviembre - diciembre • 2014 " " " " " " " " " " -

\section{Resolución de las crisis bancarias: el mecanismo de resolución único (MRU)}

El mecanismo de resolución único actúa mediante un consejo de resolución y tendrá a su disposición un fondo de resolución para salvar o liquidar a un banco. El mecanismo sólo entrará en aplicación si un banco está a punto de quebrar o si quiebra y en ambos casos será el consejo de resolución único el que deberá intervenir y encargarse de los procedimientos, y dar una respuesta en un período de tiempo mínimo. Esta directiva fue adoptada en diciembre del año 2013 por la Comisión Europea, los ministros de finanzas y el Parlamento Europeo, sin embargo el MRU entrará en vigor en el año 2016.

Se aprobó, en esta tesitura, la creación de un fondo de resolución europeo financiado por el sector bancario, el cual va a usarse cuando la contribución de los accionarios y de los prestamistas no sea suficiente; este fondo se aprovisionará cada año solamente con los recursos de los bancos. Cada banco comercial va a hacer una contribución al mismo fondo en función de su tamaño y de su perfil de riesgo. Todos los bancos comerciales deberán hacer aportaciones en función de sus indicadores de riesgo y habrá un esquema especial de contribución para los bancos muy pequeños.

El banco en dificultades financieras gozará de un apoyo a mediano plazo para poder restructurarse si las pruebas de estrés indican que tiene un cierto nivel de solvencia. Sin embargo, este fondo de la Zona Euro se va a constituir poco a poco en un período de 8 años que comienza en el año 2016. El monto total acumulado en este fondo europeo va a dividirse entre los países de la Zona Euro, y así se crearán fondos nacionales de resolución.

Pero si un banco está a punto de quebrar se seguirá el mismo modelo de recapitalización interna seguido recientemente por los bancos de Chipre. A partir del año 2016 serán los accionistas y grandes ahorradores quienes tengan la obligación de recapitalizar el banco por un monto de $8 \%$ de las pérdidas del banco antes de que intervenga el fondo nacional de resolución. Esta medida puede afectar aún más a los bancos comerciales europeos de los países que todavía no salen de la crisis. Tanto España como Italia están pidiendo que dicha medida sea aplicada hasta el año 2019 en lugar del año 2016 como ha sido previsto.

El fondo de resolución europeo contará con un capital de aproximadamente 55 mil millones de euros. Algunos datos importantes a considerar son que el rescate de los bancos irlandeses costó 67500 millones de euros en el año 2010 y en el año 2012 el rescate de los bancos españoles costó 100000 
millones de euros. Por lo tanto, el dinero que se junte con este fondo de resolución europeo se piensa que es poco e insuficiente todavía.

\section{Nuevo sistema armonizado de garantía de los depósitos}

La Comisión Europea vio la necesidad de revisar a fondo la directiva sobre la garantía de los depósitos, dado que ésta ya existía en la Unión Europea pero funcionaba de forma muy diferente entre los propios países de la Unión: antes de la más reciente crisis financiera el sistema de garantía de depósitos aseguraba a los ahorradores por 20000 euros, pero con la crisis este monto pasó a 50000 euros y había algunas excepciones de países como Francia donde este monto llegó a ser de 70000 euros.

La directiva 2014/49/ue que implementa un nuevo sistema de garantía de depósitos fue adoptada definitivamente el 16 de abril de 2014 y es a partir de esta fecha que los países de la Unión Europea tendrán 12 meses para adoptar esta directiva en su legislación nacional. Además esta directiva da a los gobiernos la autoridad necesaria para mejorar la administración y el control del fondo de garantía de depósitos nacional.

Con esta directiva se busca tener una armonización y simplificación del sistema de garantía de depósitos y se crean fondos nacionales de garantía de depósitos. Se pensó en este nuevo sistema para ya no tener que recurrir a utilizar el dinero de algún fondo público o el dinero de los depositantes en caso de quiebra de un banco.

La directiva sobre la garantía de los depósitos respalda que los depósitos bancarios de los ahorradores queden protegidos hasta un monto máximo de 100000 euros en el caso de que el banco quiebre. Así los ahorradores van a poder recuperar sus depósitos aún si el banco quiebra en un plazo de 7 días hábiles, mientras que anteriormente eran 20 días. También se protegerán los depósitos mayores a 100000 euros si ingresa dinero a una cuenta bancaria como resultado de la venta de una casa. Esta directiva entrará en vigor el $1^{\circ}$ de julio de 2015.

Otros aspectos que considera este nuevo sistema de garantía de depósitos son los siguientes:

- mejorar las formalidades administrativas transfronterizas de tal forma que los clientes de una sucursal situada en algún país miembro de la Unión Europea puedan ser rembolsados por el fondo de garantía de este país y no por el fondo de garantía del país de origen del banco. 
Economía Informa núm. 389 noviembre - diciembre • 2014 " " " " " " " " " " -

- todas las cuentas denominadas en otras divisas son cubiertas por la garantía de depósitos

- mejorar la información que los bancos dan a sus clientes

- ante la quiebra de un banco, si no llega a tener el dinero suficiente el fondo de garantía de depósitos nacional para sacar adelante al banco, podrá pedir prestado un monto de dinero limitado ante otros sistemas de garantía depósitos de la Unión Europea.

Esta directiva sobre la garantía de los depósitos forma parte de un esquema más amplio a nivel de la Unión Europea donde se aplicará un reglamento uniforme a los 8300 bancos de la Unión Europea -6 000 de la Zona Euro- que consiste en leyes y reglas con respecto a un mayor nivel de capitalización de los bancos y reglas que garanticen un mejor control de los riesgos.

El BCE y el Parlamento Europeo mantienen un diálogo en materia de política monetaria, sin embargo el $\mathrm{BCE}$ es totalmente independiente en cuanto a sus decisiones. Ahora que el BCE ha asumido mediante la Unión Bancaria Europea nuevas funciones de supervisión deberá entregar un reporte anual al Parlamento Europeo.

\section{Conclusiones}

No solamente la Unión Europea tuvo que restructurar su esquema institucional de supervisión a raíz de la crisis financiera, también a partir del año 2010 en Estados Unidos se crea el Comité de Supervisión de la Estabilidad Financiera (FSOC) que identifica los riesgos sistémicos y tiene la obligación de supervisar a los bancos y a las sociedades financieras no bancarias. Asimismo, Inglaterra también tuvo que reorganizar su sistema de supervisión el cual anteriormente estaba a cargo de la Financial Service Authority. En abril del año 2013, desaparece esta institución y a partir de esta fecha el Banco de Inglaterra asume la totalidad de las funciones de supervisión financiera.

Hay una estrecha relación entre los niveles de deuda del año 2013 y los resultados de las pruebas de estrés, como puede observarse en la gráfica 1 sobre la deuda total gubernamental por país del año 2013, los 10 países con mayores niveles de deuda, con excepción de España, fueron los que tuvieron resultados negativos en las pruebas de estrés realizadas a una pequeña muestra de bancos comerciales en cada país de la Zona Euro a principios del año 2014 


\section{Gráfica I}

Deuda total del gobierno del año 2013, por país

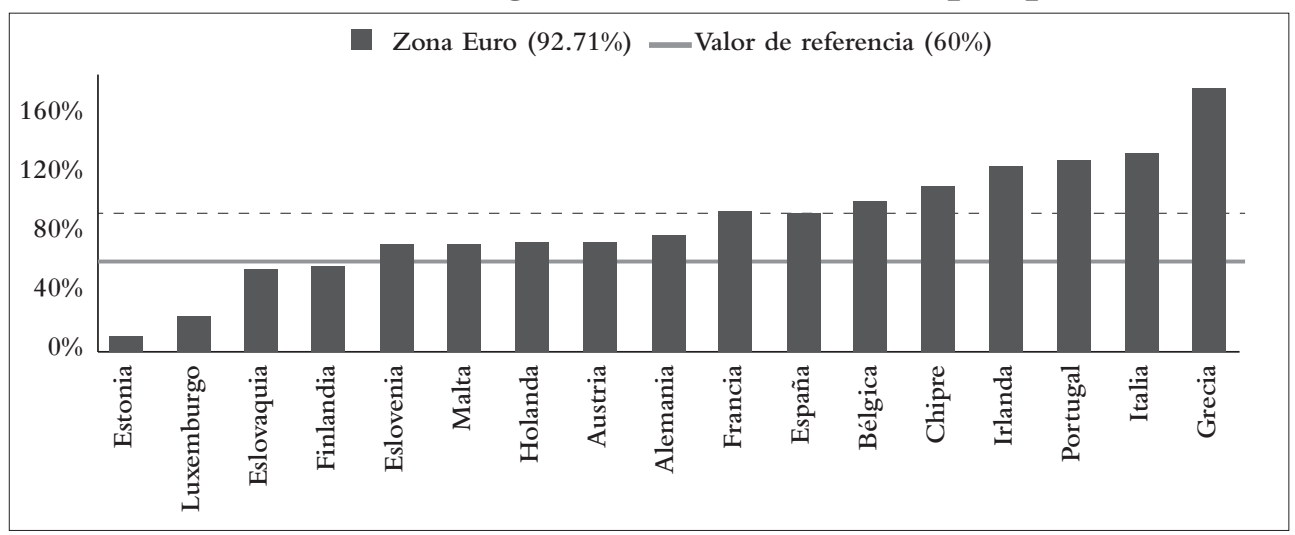

Fuente: https://www.ecb.europa.eu/stats/gov/html/dashboard.en.html.

Es importante destacar que la totalidad de las funciones de la Unión Bancaria entrarán en vigor en diferentes fechas: la función de supervisión inició el 4 de noviembre de 2014, mientras que el sistema de garantía de depósitos entrará en aplicación a mediados del año 2015 y el fondo de resolución único entrará en vigor hasta el año 2016. Hay una gran urgencia de que el conjunto de funciones de la Unión Bancaria entre en vigor, dada la quiebra del banco portugués Espirito Santo en el año 2014.

\section{Bibliografía}

Cérisier, Frederique (2014), "Union Bancaire: le champs des possibles", Conjoncture, BNP Paribas, abril.

Charrel Marie (2014), "Vingt-cinq banques européennes ont échoué aux test de résistance de la ВСЕ", Le Monde, 6 de noviembre.

Crédit Agricole (2014), “все: les questions clés en 2014”, Etudes Economiques.

Creel Jerome et Hubert Paul (2014), "Vers une meilleure gouvernance dans l’Union Européenne? La BCE ou comment devenir moins conventionnel”, Blog OFCE, 9 de abril.

Commission Européenne (2014), "L’Union Bancaire".

Sitio de internet: http://ec.europa.eu/internal_market/finances/banking-union/ index_fr.htm.

ECB (2014), "Supervision Bancaire: Le Mécanisme de Surveillance Unique”, Le Marché Unique de l’UE, Rubrique Services Financiers. 
Economía Informa núm. 389 noviembre - diciembre • 2014 ㅁ

Sitio de internet: https://www.ecb.europa.eu/ssm/html/index.fr.html.

ECB (2014), "L'examen approfondi mené par la BCE montre que les banques doivent prendre des mesures supplémentaires", Comunicado de Prensa, 26 de octubre.

Sitio internet: https://www.ecb.europa.eu/press/pr/date/2014/html/pr141026. fr.html

El Economista (2014), "Europa adopta Unión Bancaria”, domingo 12 de octubre.

Didier Raphael (2014), "Les stress test sont-ils un gage de solidité du système bancaire européen?”, Le Monde, 28 de octubre.

Michel Anne (2014), "Banques: l’Union Européenne débute son opération vérité", $1^{\circ}$ de mayo, Journal Le Monde.

Ministère de l'Economie et des Finances (2014), "Une étape supplémentaire vers l'Union Bancaire", Section Europe et International, 16 de abril.

Sitio internet de la Secretaría de Economía de Francia: http://www.economie.gouv. fr/europe-union-bancaire

Ministère de l'Economie et des Finances (2014), "Union Bancaire une étape majeure est franchie" Section Europe et International, 4 de noviembre.

Sitio internet Secretaría de Economía de Francia: http://www.economie.gouv.fr/ union-bancaire-une-etape-majeure-est-franchie

Natixis (2013), "Union Bancaire: Un point d'étape", Flash Economie, 23 de mayo.

Parlamento Europeo (2014), "El Parlamento Europeo da el paso definitivo hacia la unión bancaria", nota de prensa del 15 de abril.

Sitio de internet: http://www.europarl.europa.eu/news/es/news-room/ 